\title{
The "collateral damage" of the war on COVID-19: impact of the pandemic on the care of epithelial ovarian cancer
}

\author{
Luxitaa Goenka ${ }^{1}$ Annuja Anandaradje ${ }^{2} \cdot$ Tejaeswar Nakka $^{1} \cdot$ Smita Kayal ${ }^{1} \cdot$ Biswajit Dubashi ${ }^{1}$. \\ Latha Chaturvedula ${ }^{3} \cdot$ Pampapati Veena $^{3} \cdot$ Jayalakshmi Durairaj $^{3} \cdot$ Prasanth Penumadu $^{4} \cdot$ Prasanth Ganesan $^{1}$ (I)
}

Received: 12 August 2021 / Accepted: 19 September 2021 / Published online: 28 September 2021

○) Springer Science+Business Media, LLC, part of Springer Nature 2021

\begin{abstract}
The covid-19 pandemic has impacted the management of non-covid-19 illnesses. Epithelial ovarian cancer (EOC) requires long-duration multidisciplinary treatment. Teleconsultation and shared care are suggested solutions to mitigate the consequences of the pandemic. However, these may be challenging to implement among patients who come from the lower economic strata. We report the disastrous impact of the pandemic on the care of EOC by comparing patients who were treated during the pandemic with those treated in the previous year. We collected the following data from newly diagnosed patients with EOC: time from diagnosis to treatment, time for completion of planned chemotherapy, and proportion of patients completing various components of therapy (surgery and chemotherapy). Patients treated between January 2019 and September 2019 (Group 1: Pre-covid) were compared with those treated between January 2020 and December 2020 (Group 2: During covid pandemic). A total of 82 patients were registered [Group 1: 43(51\%) Group 2: 39(49)]. The median time from diagnosis to start of treatment was longer in group 2 when compared to group 1 [31(23-58) days versus $17(11-30)$ days $(p=0.03)$ ]. The proportion of patients who had surgery in group 2 was lower in comparison to group 1 [33(77\%) versus $21(54 \%)(p=0.02)$ ]. Proportion of patients who underwent neoadjuvant (NACT) and surgery were fewer in group 2 in comparison to group 1 [9(33\%) versus $18(64 \%) p=0.002$ ]. Among patients planned for adjuvant chemotherapy, the median time from diagnosis to treatment was longer in group 2 [28(17-45) days, group 1 versus 49(26-78) days, group $2(p=0.04)]$. The treatment of patients with EOC was adversely impacted due to the COVID-19 pandemic. There was a compromise in the proportion of patients completing planned therapy. Even among those who completed the treatment, there were considerable delays when compared with the pre-covid period. The impact of these compromises on the outcomes will be known with longer follow-up.
\end{abstract}

Keywords COVID-19 pandemic $\cdot$ Epithelial ovarian cancer $\cdot$ Treatment delay $\cdot$ National lockdown

\section{Introduction}

Epithelial ovarian cancer (EOC) is one of the commonest cancers among women and is associated with the highest mortality. Most of these women present in advanced stages

Prasanth Ganesan

pg1980@gmail.com; p.ganesan@jipmer.edu.in

1 Department of Medical Oncology, JIPMER, Dhanvantri Nagar, Puducherry 605006, India

2 Department of Clinical Pharmacology, JIPMER, Puducherry, India

3 Department of Obstetrics \& Gynecology, JIPMER, Puducherry, India

4 Department of Surgical Oncology, JIPMER, Puducherry, India and require multi-modal cancer therapy, which extends over months involving surgery and chemotherapy [1]. It has been recognized that the coronavirus disease 2019 (COVID-19) pandemic has disrupted cancer care delivery [2], [3]. The impact has been due to two reasons: there has been a partial or complete shutdown of non-covid services in hospitals due to the overwhelming load of patients with COVID-19 illness and the "lockdown," which was essential to control the spread of infection in society, affecting the movement of patients to the hospital [4].

At our center, a major tertiary center in South India and a designated COVID-19 care hospital, the oncology services were constrained with partial or complete shutdown during the peak of the "first wave" of the pandemic in 2020. Even though cancer care services (including chemotherapy and surgery) were prioritized when compared to other non-covid 
illnesses, compromise in care was inevitable in the disaster situation. Our center caters to the poorest of the society from the states of Puducherry and the districts of Tamilnadu, with most patients depending on public transport to reach the hospital for care. Thus the "lockdown" made it difficult for patients to attend the hospital for treatment. To understand the exact impact of the pandemic on the care of $\mathrm{EOC}$ at our center, we compared patients with EOC who were treated during the pandemic with those treated in the previous year.

\section{Methods}

\section{Study design and data collection}

We retrospectively collected data from patients diagnosed with EOC registered at our department during two separate periods: those treated between 1st January 2019 and 30th September 2019 (Group 1: Pre-covid group) and those treated between 1st January 2020 and 31 st December 2020, who were expected to be maximally affected by the pandemic (Group 2: During-covid group). Group 1 was chosen such that it would be expected that they would have completed most of their treatment before the onset of the pandemic (assuming a six-month time frame to complete the treatment of EOC and 1st March 2020, as the start of pandemic-induced disruption in services). Data regarding baseline characteristics [age, grade, stage, Eastern Cooperative Oncology Group (ECOG) performance status (PS), and comorbidities] and therapeutic details [surgery and chemotherapy] were collected. Data on timelines such as time from diagnosis to start of treatment, time from the start of chemotherapy to surgery in case of neoadjuvant chemotherapy (NACT), the total time for completion of treatment (surgery and chemotherapy), and time between first and last cycle of chemotherapy were collected.

These were patients registered for the treatment of EOC at our center. The usual care of these patients included regular phone calls to those not following up for their planned treatment or follow up visits. In addition, during the pandemic period, regular teleconsultation services were provided for all patients. Those who couldn't travel to our center were encouraged to take treatment at local hospitals and references to oncologists who could provide these services nearer to the patient's residence were provided.

\section{Analysis}

Continuous variables were summarized as the mean \pm standard deviation or median (IQR), and categorical data were expressed as frequency and percentages. The differences between groups were analyzed using the Mann-Whitney
U test. Differences in the categorical variables were analyzed using the chi-square test. Statistical analyses were performed using SPSS software version 19.0 (SPSS Inc., Chicago, IL, USA). All values were two-sided, and a value of $p<0.05$ was considered statistically significant. For the purpose of analysis, the patients were separately analyzed based on whether the initial intent of treatment was upfront surgery, or those who underwent NACT with intent for surgery later.

\section{Results}

A total of 82 patients were registered [Group 1: $43(51 \%)$ Group 2: 39 (49\%); median age: 54 (48-57) years and 55 (47-65) years]. Majority of the patients were in either stage III/IV in both the groups [Group 1: $n=37(86 \%)$ ] and [Group 2: $n=33(85 \%)$ ] (Table 1 represents the baseline characteristics of the patients).

Considering all patients, the median time from diagnosis to start of treatment was longer in group 2 when compared to group 1 [31 (23-58) days versus $17(11-30)$ days $(\mathrm{p}=0.03)$ ]. A significantly lesser number of patients in group 2 underwent surgery when compared to group 1 [33 (77\%) versus $21(54 \%)(p=0.02)]$.

\section{Patients planned for NACT}

Among the 55 patients scheduled for NACT (group 1: $N=28$ and group 2: $N=27$ ), the median time from diagnosis to treatment was similar between group 1 and group 2 [17 (11-27) days and 18 (9-37) days, respectively]. In group 1 , among the 28 patients who started NACT, 18/28 (64\%) underwent surgery at a median time of 122 (93-158) days from the start of chemotherapy. In group 2, among the 27 patients who started chemotherapy, only 9 underwent surgery after a median time of 121 (91-271) days from the start of chemotherapy. Thus, the proportion of patients undergoing surgery was significantly lower in group 2 when compared to group 1 [9/27 (33\%) versus 18/28 (64\%) $p=0.002]$ (Table 2).

The proportion of patients completing six cycles of chemotherapy was 22/28 (78\%) in group 1 and 15/27 (57\%) in group 2. However, among those who completed their chemotherapy courses, the time to completion was not different between the two groups [group 1: 167 (146-229) days vs. group 2: 147 (126-198) days].

\section{Patients planned for upfront surgery and then planned for adjuvant treatment}

In these patients, the median time from diagnosis to treatment was longer in group 2 when compared to group 1 
Table 1 Baseline characteristics of study patients

\begin{tabular}{|c|c|c|c|c|}
\hline Sl No & Characteristics & $\begin{array}{l}\text { Group } 1 \text { (Pre-COVID) } \\
(N=43)\end{array}$ & $\begin{array}{l}\text { Group } 2 \text { (During } \\
\text { COVID) }(N=39)\end{array}$ & $P$ value \\
\hline 1 & Age, years & $54(48-57)$ & $55(47-65)$ & 0.30 \\
\hline \multirow[t]{5}{*}{2} & Grade, [n (\%)] & & & \\
\hline & High & $38(88 \%)$ & $30(77 \%)$ & 0.51 \\
\hline & Low & $4(9 \%)$ & - & \\
\hline & Borderline & $1(3 \%)$ & $3(8 \%)$ & \\
\hline & Unknown & - & $6(15 \%)$ & \\
\hline \multirow[t]{3}{*}{3} & Stage, $[\mathrm{n}(\%)]$ & & & \\
\hline & I, II & $6(14 \%)$ & $6(15 \%)$ & 0.85 \\
\hline & III, IV & $37(86 \%)$ & $33(85 \%)$ & \\
\hline \multirow[t]{3}{*}{4} & ECOG, $[\mathrm{n}(\%)]$ & & & \\
\hline & 0,1 & $19(44 \%)$ & $19(49 \%)$ & 0.68 \\
\hline & 2,3 & $24(56 \%)$ & $20(51 \%)$ & \\
\hline \multirow[t]{4}{*}{5} & Comorbidities, [n (\%)] & & & \\
\hline & Diabetes & $2(5 \%)$ & $16(41 \%)$ & 0.0001 \\
\hline & Hypertension & $5(12 \%)$ & $10(26 \%)$ & 0.10 \\
\hline & Post-menopausal & $32(74 \%)$ & $36(92 \%)$ & 0.03 \\
\hline
\end{tabular}

ECOG Eastern Cooperative Oncology Group

Table 2 Impact of COVID-19 lockdown on treatment delays

\begin{tabular}{|c|c|c|c|c|}
\hline S1 No & Characteristics & $\begin{array}{l}\text { Group } 1 \\
\text { (Pre-COVID) } \\
(N=43)\end{array}$ & $\begin{array}{l}\text { Group } 2 \text { (During } \\
\text { COVID) }(N=39)\end{array}$ & $P$ value \\
\hline 1 & Time from diagnosis to start of treatment & $17(11-30)$ & $31(23-58)$ & $\mathbf{0 . 0 3}$ \\
\hline 2 & Proportion of patients who had surgery & $33(77 \%)$ & $21(54 \%)$ & 0.02 \\
\hline \multirow[t]{9}{*}{3} & NACT patients & & & \\
\hline & Time from diagnosis to start of treatment & $17(11-27)$ & $18(9-37)$ & 0.93 \\
\hline & Number who started NACT & $28(51 \%)$ & $27(49 \%)$ & \\
\hline & Number who underwent surgery after NACT & $18(64 \%)$ & $9(33 \%)$ & $\mathbf{0 . 0 2}$ \\
\hline & Time between first and last cycle of chemotherapy & $159(125-211)$ & $116(48-152)$ & 0.004 \\
\hline & Time to surgery from start of chemotherapy (among those who underwent surgery) & $122(93-158)$ & $121(91-271)$ & 0.93 \\
\hline & Total time for completion of treatment & $159(125-212)$ & $116(45-177)$ & 0.01 \\
\hline & Number of patients who completed all 6 cycles of chemotherapy & $22(78 \%)$ & $15(57 \%)$ & 0.09 \\
\hline & $\begin{array}{l}\text { Total time for completion of treatment among those who completed } 6 \text { cycles of chemo- } \\
\text { therapy }\end{array}$ & $167(146-229)$ & $147(126-198)$ & 0.36 \\
\hline \multirow[t]{7}{*}{4} & Patients who were planned for upfront surgery & & & \\
\hline & Time from diagnosis to start of treatment & $28(17-45)$ & $49(26-78)$ & 0.04 \\
\hline & Number who underwent surgery & $15(56 \%)$ & $12(44 \%)$ & \\
\hline & Time between first and last chemotherapy & $109(107-130)$ & $86(31-211)$ & 0.40 \\
\hline & Total time for completion of treatment & $151(135-181)$ & $96(57-230)$ & 0.33 \\
\hline & Number of patients who completed all 6 cycles of chemotherapy & $13(86 \%)$ & $3(27 \%)$ & 0.002 \\
\hline & $\begin{array}{l}\text { Total time for completion of treatment among those who completed } 6 \text { cycles of chemo- } \\
\text { therapy }\end{array}$ & $151(139-197)$ & $182(173-238)$ & 0.14 \\
\hline
\end{tabular}

NACT Neoadjuvant chemotherapy

[49 (26-78) days versus $28(17-45)$ days $(p=0.04)$ ]. The proportion of patients who had completed all six cycles of chemotherapy were lesser in group 2 in comparison to group 1 [ $3(27 \%)$ versus $13(86 \%) ; p=0.002$ ].
(Tables 2, 3). However, among those who were able to complete all six courses, the time between the first and last cycle of chemotherapy was similar in both the groups 
Table 3 Status of delivery of surgery and chemotherapy during COVID-19 (group 2)

\begin{tabular}{lc}
\hline Characteristics & $\begin{array}{l}\text { Group 2 (Dur- } \\
\text { ing COVID-19) } \\
\text { (N=39) }\end{array}$ \\
\hline Surgery details & $2(5 \%)$ \\
Surgery not planned & \\
Surgery planned and conducted as planned & $10(26 \%)$ \\
Surgery planned but delayed & $9(23 \%)$ \\
Surgery was planned but not done & $18(46 \%)$ \\
Chemotherapy details & \\
Continued without any interruption & $17(43 \%)$ \\
Interrupted & $12(32 \%)$ \\
Interrupted but restarted & $4(10 \%)$ \\
No chemo planned & $1(3 \%)$ \\
Switched to some other form like oral medica- & $5(12 \%)$ \\
$\quad$ tions & \\
\hline
\end{tabular}

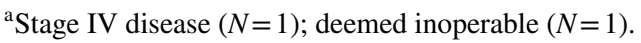

${ }^{\mathrm{b}}$ Surgery was part of the treatment plan; however, because of covid19 , the surgery was postponed as the theaters were closed in our center and the patients did not undergo surgery at any other center.

of patients [Group 1: 109 (107-130) days versus Group 2: $86(31-211)$ days $(p=0.40)]$.

\section{The overall impact of the Covid-19 pandemic on the care of women with ovarian cancers}

None of the patients in group 2 were diagnosed with Covid-19 infection during the period of observation. Among the 43 patients treated during the Covid19 pandemic (group 2), 38 were planned for surgery (5 were unfit due to extensive disease). However, due to the lockdown effect on the delivery of surgical services, surgery was not done in 18/38 (49\%) patients, among whom it was part of the initial treatment plan. This was because the operation theaters were closed or because the patients could not travel to the center due to the lockdown. Although the impact on chemotherapy was lesser, only 17 patients completed planned chemotherapy without interruptions (43\%). In all other patients, there was some compromise in the chemotherapy, including complete interruption $(N=12,32 \%)$, breaks but restarted and completed $(N=4,10 \%)$, and switch to oral chemotherapy $(N=5,12 \%)$ (Table 3). Even though some patients were contacted through telemedicine and were encouraged to complete their treatment at another center, none of the 39 patients either took chemotherapy or underwent surgery at a different center.

\section{Discussion}

Non-covid medical services, especially cancer care, have been badly affected during the COVID-19 pandemic [3]. The impact may be variable depending on the type of cancer (a requirement for multidisciplinary care) and the socioeconomic profile of the patients. Although there are papers reporting the overall impact, the exact extent of the compromise in individual cancers is unknown. EOC requires complex management with surgery and chemotherapy and takes about six months to complete full treatment [5]. Such patients are likely to be severely impacted in the context of the pandemic-induced disruption of hospital services, and lockdown-related travel restrictions. We present an audit which reveals the disastrous impact of the pandemic on the care of EOC. Nearly half the patients did not undergo planned surgery and chemotherapy. Although teleconsultation and referral to nearby centers for treatment completion have been put forth as mechanisms to mitigate the consequences of the pandemic, our experience reveals that, in reality, neither of these are helpful. Although many patients were encouraged to consider undergoing surgery at other centers which may have been offering these services and appropriate references were provided, they refused/ or couldn't go elsewhere. This could be because of the low socioeconomic status of patients who visit our center, and hefty charges for cancer care in private centers would impose a financial burden on them. Furthermore, some patients travel long distances for therapy, necessitating the search for lodging and food. Obtaining food and shelter during the lockdown is another major challenge [6]. Many of these patients have since suffered progression of the disease and had returned to our center and restarted therapy (however, this data is not captured for this paper).

A large multi-institutional study from India [3] showed a $49 \%$ reduction in surgical rates than pre-pandemic times. Our analysis confirms that this 50\% drop in services is not compensated by patients completing their treatment at another center. Similar studies from other parts of the world detail the impact of COVID-19 [7]. Other indicators of loss of care during the pandemic include a reduction in applications for insurance support (cancer-related applications reduced by $64 \%$, according to data from Prime Minister Jeevandayee Arogya Yojana, major government sponsored insurance scheme helping patients in India), overwhelmed system, lack of personal protective equipment, shortage of manpower, and restricted access to medications [8] [9].

One center in India implemented priority services for certain cancers and delivered some of the services optimally [10]. However, this was a private center, possibly 
catering to a profile of patients from a higher socioeconomic stratum. Moreover, one-third of the patients undergoing curative-intent treatment had compromised care even in their report. According to a recent Markov modelbased estimate of lifetime health outcomes among women with cervical cancer in India, mortality will increase from 2.52 percent to 3.80 percent due to pandemic [11]. Similarly, inevitably, compromised care delivered to patients with EOC during the pandemic will adversely affect their outcomes. Considering that the mortality of EOC is very high $(60 \%-70 \%)$, even with the optimally delivered care, the impact of the compromised care will be devastating [12].

Two causes may be there for the compromised care: one is the disruption of services in hospitals designated for covid care, while the other is because of the lockdown effect. While the latter cannot be easily tackled when there is an overwhelming pandemic situation, the former can be partly addressed. Efforts must be made by tertiary centers dealing with poor patients not to stop advanced care during pandemic crises [13]. It is obvious that these patients are unable to obtain specialized services from other centers. Instead, efforts must be made to distribute covid care services to all hospitals so that tertiary centers can continue to deliver specialized care. Otherwise, these patients are rendered as "collateral damage" during our fight against COVID-19, and are not counted toward the mortality burden imposed by the pandemic.

Author contributions LG contributed to acquisition, analysis, or interpretation of data for the work; drafting of the manuscript; final approval of the version to be published. AA and TN contributed to acquisition of data for the work; revising the manuscript critically for important intellectual content; final approval of the version to be published. SK, $\mathrm{BD}, \mathrm{LC}, \mathrm{PV}, \mathrm{JD}$, and PP contributed to revising the manuscript critically for important intellectual content and final approval of the version to be published. PG contributed to conception or design of the work analysis or interpretation of data for the work; revising the manuscript critically for important intellectual content; drafting of the manuscript; final approval of the version to be published; agreement to be accountable for all aspects of the work in ensuring that questions related to the accuracy or integrity of any part of the work are appropriately investigated and resolved.

Funding The study was supported by JIPMER. No grant number is applicable.

\section{Declarations}

Conflict of interest None of the authors have any relevant conflict of interest to declare.

\section{References}

1. Ferlay J, Soerjomataram I, Dikshit R, Eser S, Mathers C, Rebelo $\mathrm{M}$, et al. Cancer incidence and mortality worldwide: sources, methods and major patterns in GLOBOCAN 2012. Int J Cancer. 2015;136(5):E359-386.

2. Shankar A, Saini D, Goyal N, Roy S, Angural H, et al. Cancer Care Delivery Challenges in India during the COVID-19 Era: Are We Prepared for the Postpandemic Shock? Asia-Pac J Oncol Nurs. 2021;8(1):1-4.

3. Ranganathan P, Sengar M, Chinnaswamy G, Agrawal G, Arumugham R, Bhatt R, et al. Impact of COVID-19 on cancer care in India: a cohort study. Lancet Oncol. 2021;22(7):970-6.

4. Disis ML. Oncology and COVID-19. JAMA. 2020;324(12):1141-2.

5. Ledermann JA, Raja FA, Fotopoulou C, Gonzalez-Martin A, Colombo N, Sessa C, et al. Newly diagnosed and relapsed epithelial ovarian carcinoma: ESMO clinical practice guidelines for diagnosis, treatment and follow-up. Ann Oncol Off J Eur Soc Med Oncol. 2018;29(Suppl 4):iv259.

6. Ghosh. Impact of Covid-19 Pandemic on the Treatment of Cancer Patients Registered at a Tertiary Care Centre in Eastern India.

7. De Vincentiis L, Carr RA, Mariani MP, Ferrara G. Cancer diagnostic rates during the 2020 "lockdown", due to COVID-19 pandemic, compared with the 2018-2019: an audit study from cellular pathology. J Clin Pathol. 2021;74(3):187-9.

8. Smith. National Health Authority - PM-JAY Policy Brief 8. PMJAY Under Lockdown: Evidence on Utilization Trends.

9. Jazieh AR, Akbulut H, Curigliano G, Rogado A, Alsharm AA, Razis ED, et al. Impact of the COVID-19 pandemic on cancer care: A global collaborative study. JCO Glob Oncol. 2020;6:1428-38.

10. Mallick I, Chakraborty S, Baral S, Saha S, Lal VH, Sasidharan R, et al. Prioritizing delivery of cancer treatment during a COVID-19 lockdown: The experience of a clinical oncology service in India. JCO Glob Oncol. 2021;7:99-107.

11. Gupta N, Chauhan AS, Prinja S, Pandey AK. Impact of COVID19 on outcomes for patients with cervical cancer in India. JCO Glob Oncol. 2021;7:716-25.

12. Torre LA, Trabert B, DeSantis CE, Miller KD, Samimi G, Runowicz CD, et al. Ovarian cancer statistics, 2018. CA Cancer J Clin. 2018;68(4):284-96.

13. Kumar J, Kumar P. COVID-19 pandemic and health-care disruptions: count the most vulnerable. Lancet Glob Health. 2021;9(6):e722-3.

Publisher's Note Springer Nature remains neutral with regard to jurisdictional claims in published maps and institutional affiliations. 